\title{
SC|A2
}

Atribuiçãa BB CY 4.0

\section{Redes femininas de apoio nas ciências exatas: Por que tão necessárias?}

\author{
Viviane Brito Nogueira ${ }^{1}$ \\ Izabela Lima Paiva ${ }^{2}$
}

\section{Resumo}

A desigualdade entre sexos na ciência perdura, especialmente na ascensão de carreira e nas ciências exatas. Esse relato de experiência explora as dificuldades e estratégias de enfrentamento na trajetória de pesquisadoras atuantes na área de exatas. Depoimentos foram coletados em formato de entrevista semiestruturada, seguido de uma avaliação do discurso. Focando nos pontos de interseção entre os depoimentos, encontrou-se: sentimento de rejeição no dia a dia; consequências na saúde mental; ausência de pesquisadoras como referência em institutos de pesquisa de computação; e como estratégia de enfrentamento tem-se redes femininas de apoio. Diante disso, conclui-se que, apesar do ambiente hostil vivenciado e aqui discutido com bases sociológicas e históricas, o espaço seguro de troca, de apoio emocional e de confiança desenvolvido nas redes de apoio femininas desempenham um papel essencial na permanência de mulheres na ciência. A função das redes se torna ainda mais relevante em meio a pandemia da COVID-19 de 2020.

\section{Palavras-chave}

Estratégias de enfrentamento. Mulheres na Ciência. Quarentena. Síndrome do impostor. STEM.

Recebido em: 30/07/2020 Aprovado em: 26/01/2021

\footnotetext{
${ }^{1}$ Biotecnologista, Mestre e Doutoranda em Ciências da Saúde. É defensora e atuante na equidade de gêneros na ciência, em educação de qualidade, e saúde e bem estar (ODS $3,4,5$ ) para todos. Realiza divulgação científica produzindo Podcast.

E-mail: vivianebritonogueiraa@gmail.com

${ }^{2}$ Farmacêutica, Mestranda no Instituto do Cérebro. Defende e atua na busca por equidade entre gêneros e na política de redução de danos no uso de drogas.

E-mail: izalpaiva@gmail.com
} 


\section{Introdução}

A desigualdade entre sexos ainda prevalece no mundo. Um estudo do Fórum Econômico Mundial contemplou a paridade entre homens e mulheres nas áreas de política, educação, trabalho e saúde e projetou a necessidade de 99,5 anos para que uma igualdade média global seja alcançada. No ranking de igualdade entre sexos, o Brasil está na $92^{\mathrm{a}}$ posição, de um total de 153 países. O estudo leva em consideração a velocidade de progressão observada entre 2006 e 2020, e faz projeções de acordo com as subáreas analisadas: para que a desigualdade de cunho profissional seja extinta serão necessários 257 anos. Adicionalmente, a diferença salarial global entre os sexos é de 40\% (WORLD ECONOMIC FORUM, 2019).

Essa desigualdade é refletida no meio acadêmico e científico. Professores de universidades com atuação intensiva em pesquisa tendem a contratar um profissional homem para seu laboratório e orientá-lo, pagar-lhe mais e classificá-lo como mais competente do que uma candidata com o mesmo currículo (MOSS-RACUSIN et al., 2012; STEINPREIS; ANDERS; RITZKE, 1999). Professores também tendem a responder mais a e-mails de estudantes homens interessados no doutorado do que de estudantes do sexo feminino (MILKMAN; AKINOLA; CHUGH, 2015). Avaliadores de solicitações de bolsas de pós-doutorado nas ciências biomédicas tendem a exigir que mulheres tenham 2,5 vezes mais produtividade que os homens para serem igualmente classificadas como cientificamente competentes (WENNERAS; WOLD, 1997). Esses estudos indicam que o nível de exigência na ciência para mulheres é mais alto do que para homens.

Para contextualizar este relato de experiência, um recorte para a área de exatas é importante. Mesmo com inúmeras contribuições científicas de mulheres ao longo da história do prêmio Nobel, elas são apenas cerca de $5 \%$ dos premiados (ORGANIZAÇÃO PRÊMIO NOBEL, 2019). Analisando as categorias, os prêmios Nobel de física, química e economia tem um número ainda menor de contempladas. Como a matemática não entra no Nobel, existem dois prêmios considerados análogos: o Prêmio Abel e a Medalha Fields. A premiação da 
medalha Fields acontece a cada 4 anos desde 1936 - a primeira e única mulher contemplada até então foi a Iraniana Maryam Mirzakhani em 2014 (INTERNATIONAL MATHEMATICAL UNION (IMU), 2018). Já o prêmio Abel acontece anualmente desde 2003 - a estadunidense Karen Uhlenbeck em 2019 também foi a primeira e única mulher contemplada (ORGANIZAÇÃO PRÊMIO ABEL, 2020). Isso aponta para uma ausência de referências de mulheres cientistas em exatas para as novas gerações.

A discrepância entre sexos aqui apresentada pode afetar a saúde mental de pesquisadoras. A síndrome do impostor descreve indivíduos de alto desempenho que, apesar do sucesso profissional, falham em internalizar suas realizações de forma persistente e sentem insegurança e/ou medo de serem expostos como uma fraude (KOLLIGIAN; STERNBERG, 1991). Frequentemente se apresenta junto a depressão e ansiedade. Está associada a prejuízos de desempenho e satisfação no trabalho e exaustão, em diversas áreas do mercado. Essa síndrome afeta os dois sexos. Alguns estudos apontam uma tendência maior em mulheres, e outros não encontram diferença significativa (BRAVATA et al., 2020). É possível haver relação com o momento da carreira: ao avaliar duas populações (estudantes e profissionais em psicologia), os efeitos de gênero para a síndrome do impostor foi significativo entre os estudantes, mas não entre profissionais (BRAUER; PROYER, 2017). Isso remete ao mecanismo social "teto de vidro", que dificulta a ascensão hierárquica de mulheres em seus ambientes de trabalho (DAVIDSON, 1992; MADALOZZO, 2011; WRIGHT; BAXTER, 2000). O mecanismo é descrito para as pessoas que estão na construção da carreira e não para os que já se encontram nos cargos de liderança (COELHO; DE NEGRI; DE NEGRI, 2006). Nesse contexto, são múltiplos os estímulos psicossociais que podem prejudicar a carreira de mulheres cientistas.

Apesar do cenário pouco favorável, pesquisadoras encontraram estratégias de resistência e permanência. Esse artigo se propõe a abordar essas estratégias e contextualizá-las na quarentena imposta devido a pandemia da COVID-19 em 2020. Ressalta-se que a produtividade de pesquisadores está comprometida devido a quarentena. $\mathrm{E}$ isso difere de acordo com conformação familiar, etnia e tempo de carreira. Em relação a submissões de artigos, mulheres negras (com ou sem filhos) e mulheres brancas com filhos foram os 
grupos cuja produtividade acadêmica foi mais afetada - especialmente mulheres no início da carreira (até 5 anos). A produtividade acadêmica de homens, especialmente os sem filhos, foi a menos afetada pela pandemia (PARENT IN SCIENCE, 2020). Diante do exposto, em formato de relato de experiência, esse trabalho objetiva investigar as dificuldades e estratégias de enfrentamento na trajetória de pesquisadoras atuantes na área de exatas - discutindo com bases sociológicas e históricas.

\section{Metodologia}

Para avaliação da importância das redes de apoio femininas formadas por pesquisadoras nas áreas das exatas (com contextualização do cenário de pandemia da COVID-19), foram coletados depoimentos de duas pesquisadoras mulheres cis gênero, atuantes na área de exatas, a nível de pós-graduação, com idade entre 20-30 anos, sem filhos no nordeste brasileiro. Para coleta dos depoimentos foi utilizado o modelo de entrevista semiestruturada (MAY, 2004). As perguntas norteadoras foram: (1) Como é ou como foi sua trajetória de atuação nas ciências exatas? e (2) A interação com outras mulheres colabora ou colaborou durante sua trajetória? Informações a mais sobre as pesquisadoras foram suprimidas a fim de preservar a identidade delas, usa-se nesse trabalho os pseudônimos: Júlia e Liz.

Os depoimentos foram coletados e processados via internet pela nuvem de armazenamento e transferência de arquivos online Google Drive. Foi realizada uma categorização de elementos presentes nos depoimentos, considerando a experiência, a vivência e a ação das participantes (MINAYO, 2012), a saber: (1) principais dificuldades encontradas; (2) percepção das interações ocorridas em seu ambiente de trabalho; e (3) estratégias de enfrentamento.

\section{Resultados}

\section{Depoimento: Relato de experiência de Júlia}

Júlia iniciou sua trajetória nas áreas exatas atuando na em empresas de engenharia, área predominantemente ocupada por homens. Assumiu espaço de 
liderança em equipes, e por vezes teve sua capacidade produtiva e intelectual colocada em dúvida, recebendo por exemplo, recusas de seu superior a lhe ensinar programação em softwares específicos porque "mulheres não sabem programar e nem matemática" ouvia que "era muito feminina para essa área", "que até fazia o trabalho bem para uma mulher". Durante esse período ainda planejava cursar uma graduação em ciências exatas, entretanto, dadas as condições da época, os ataques a sua capacidade intelectual diários apenas por ser mulher e a falta de uma rede de apoio e de conhecimentos sobre como enfrentar essa situação decidiu migrar de área.

Migrou para um curso da área de ciências da saúde, onde logo de início percebeu um ambiente diferente, onde as mulheres tinham espaço de fala e ocupavam com mais frequência posições de liderança. Logo no primeiro período ingressou como aluna de iniciação científica (IC) em um laboratório com temática voltada para esta nova área e não percebia ou sentia diferenças entre ela e seus colegas.

Em dado momento, interessou-se por pesquisas com objeto de estudo mais próximo às ciências exatas. Para isso, buscou aprimorar seus conhecimentos sobre matemática, física e programação. Sentiu inicialmente o impacto e a diferença, neste novo ambiente percebeu a ausência de mulheres em posições de liderança. Nas disciplinas que cursou para aprender a programar ouvia de seus colegas assertivas como "você até que escreveu o código bem para uma mulher". Por muitas vezes se sentiu invisibilizada e sem espaço de fala. Sentindo-se por vezes incompetente e uma farsa, incapaz de assimilar tão aprendizado, passando a apresentar quadros de ansiedade.

Diante da perspectiva pensou em desistir e regressar às áreas de ciências da saúde onde sentia mais confiança. Entretanto, redes de apoio e comunicação com outras mulheres, também nas áreas exatas, trouxeram uma perspectiva nova fazendo com que a sua permanência fosse possível. Ingressou em um mestrado na área de análises de dados e engajou-se no estudo de pautas feministas. Hoje busca fortalecer as redes de apoio que a trouxeram até aqui, bem como formar uma rede de apoio para outras mulheres que cheguem a esse 
espaço. Relata que durante a pandemia da COVID-19 por meio de reuniões via plataformas digitais ou mensagens trocadas por aplicativos de mensagens essa rede de apoio tem sido de extrema importância para manutenção do interesse, da capacidade de trabalho e do bem estar mental.

\section{Depoimento: Relato de experiência de Liz}

Liz é formada na área de ciências biológicas. Ela começou a iniciação científica (IC) no primeiro semestre da graduação. Completou três projetos de IC e interrompeu o quarto para cursar um ano fora do Brasil pelo Ciência sem Fronteiras. No exterior vivenciou laboratórios de pesquisa tanto no meio acadêmico como na indústria. No retorno ao Brasil, recebeu prêmios científicos internacionais. Trabalhou como Desenvolvedora Técnico-Industrial num instituto de pesquisa. Durante todos esses anos, nunca sentiu que ser mulher a fazia diferente dos seus companheiros de trabalho. O cenário começou a mudar quando resolveu aprender a fazer análise dos dados biológicos que produzia. Afinal, analisar dados requer programação, e programar é adentrar a área de exatas. Nesse microcosmo a presença do machismo ainda é muito forte. Durante seu mestrado, fazendo a ponte entre biológicas e exatas, percebeu que a autossabotagem passou a dominá-la. Liz não queira mais ir ao seu ambiente de trabalho, sentia constante vontade de desistir.

Aqui é necessário avaliar o cenário: $\mathrm{O}$ instituto de pesquisa nesse período tinha apenas uma outra pós-graduanda mulher, que não fazia parte do seu grupo de pesquisa. Não existiam orientadoras mulheres trabalhando com análise de dados. Comentários como "Você está programando bem para uma mulher", "Não tem como ser bonita e ser da computação" ou "Não pode engravidar até terminar o doutorado" faziam parte do seu dia a dia.

Sua estratégia de enfrentamento foi estudar sobre o tema para confrontar os comentários e lidar com a rejeição que percebia no seu ambiente de trabalho. A partir disso, passou a se posicionar diariamente no instituto e a virar uma referência para os pós-graduandos homens sobre o que "poderia ou não ser dito" - cargo esse muito cansativo. Com a entrada de novas mulheres no instituto, percebeu que sua experiência não era individual, e sim amplamente 
compartilhada. A problemática está na dificuldade de expressar questões tão delicadas. Notou sua tendência a trabalhar muito - trabalhar mais do que o necessário, mais do que os homens - para conseguir se inserir e autoafirmar naquele ambiente. Isso contribuiu para o desenvolvimento de um transtorno de ansiedade generalizada. Seu tratamento é, até hoje, sua rede de apoio feminina. Essa rede é construída através de (1) encontros para partilha dentro e fora do ambiente de trabalho, (2) suas participações em eventos científicos feito por mulheres e para mulheres e (3) sua busca incessante em encontrar referências de pesquisadoras na área, seguindo-as nas redes sociais para alimentar inspiração.

Durante a pandemia da COVID-19 e a obrigação do isolamento social, compreende mais ainda a necessidade dessa rede de apoio. Cursando seu doutorado, criou junto a outra doutoranda um clube de discussão científica semanal, fazendo uso de plataformas de videoconferência com compartilhamento de tela. Isso ajuda a não perder a partilha "dentro do ambiente de trabalho". Está participando de eventos científicos virtuais entre mulheres e dá especial atenção aos posicionamentos de pesquisadoras nas redes sociais. Liz afirma que a manutenção dessa rede é essencial para sua permanência no meio acadêmico.

\section{Discussão}

Ao considerar as categorizações, observou-se nos depoimentos que (1) as principais dificuldades relatadas são a presença de sensações de rejeição, insegurança quanto a capacidade de realizações de tarefas, e o desenvolvimento de transtornos psicológicos (ansiedade generalizada e síndrome do impostor); (2) as pesquisadoras sentem o impacto da dificuldade de se manter na área e consequentemente ascender na carreira, sem encontrar referências de mulheres na liderança; e (3) como estratégia de enfrentamento é observada a formação de redes de apoio femininas, com compartilhamento dos obstáculos vivenciados, resultando no aumento da capacidade de enfrentamento e por consequência a permanência na área de ciências exatas. Essa estratégia de enfrentamento continua relevante durante a pandemia de COVID-19, onde as redes de apoio 
mostram-se cruciais para a manutenção da produtividade e do bem estar mental. Logo, esses três temas serão abordados nas subseções dessa discussão.

\section{Rejeições do dia a dia e consequências na saúde mental}

A dificuldade em se manter na área de exatas, segundo o relato das pesquisadoras, não se dá por eventos marcantes nem pela não-aceitação direta. A dificuldade se dá em meio a rejeições pontuais, e até pequenas, mas com frequência alta. É um estímulo constante na experiência de ambas. Para compreender como um ambiente pode se tornar hostil para um determinado grupo de indivíduos, os conceitos de Capital, Campo, Habitus e Violência simbólica de Pierre Bourdieu são úteis.

Capital corresponde aos recursos que uma pessoa possui e que a equipa com privilégios e vantagens em comparação com outras pessoas que não os possuem. Esses capitais podem ser econômicos, culturais, sociais ou simbólicos. O capital econômico são os recursos financeiros disponíveis, seja em dinheiro ou bens materiais. Já o capital cultural tem relação com recursos como conhecimento de livros, de oratória, ou diplomas. Capital social são os contatos que uma pessoa possui, numa rede de relacionamento, que lhe confere vantagens frente aos que não tem, e o capital simbólico é relativo à imagem, a beleza ou o status.

Campo é um espaço específico, com configuração específica, que requer de um indivíduo capitais diferentes (é uma rede ou uma configuração de relações sociais que são organizadas em posições de dominância diferentes). Ou seja, é no campo que ocorrem as disputas de poder e posição na realidade social. Dois extremos surgem dessa distribuição de poder: dominantes e dominados. Os primeiros são os que possuem mais capital específico e os outros possuem menos capital. Já Habitus diz respeito a como as pessoas aprendem e reproduzem aquilo que aprenderam na sociedade, assumindo pensamentos de sua época. Em outras palavras: como percebem o mundo e atuam nele. O habitus é a experiência social incorporada na mente - sempre construído em um indivíduo dentro de um campo, detendo alguns capitais (CORTÉS, 2016). 
O campo no contexto dos relatos de experiência é o campo científico, mais especificamente o microcosmo de institutos de pesquisa que realizam ciência de dados - predominantemente compostos por homens. O hábito mental (habitus) desse campo é uma reprodução de não-aceitação de mulheres devido sua ausência histórica. É importante ressaltar que o capital cultural dos homens tende a ser mais estimulado desde a infância o que propicia a ocupação desses espaços. São muitos os fatores socioculturais que apontam para isso. $\mathrm{O}$ desenvolvimento de gostos e habilidades, amplamente influenciados pelo ambiente (RISSO, 2008), em meio a divisão de brinquedos entre os sexos: meninas brincam prioritariamente dentro da esfera casa-cuidados (bonecas e casinha) e os meninos brincam com estímulos tridimensionais (jogos de encaixe e vídeo games) (BARBOSA; LIMA, 2013). Adicionalmente, ressalta-se que o habitus que rege as ciências exatas estão alocados no polo masculino dentro de uma binaridade: Razão, objetividade e competitividade; onde o feminino é construído como oposto: Sentimento, dedicação, minúcia e paciência (BRUSCHINI, 1988). Dessa forma, no campo de disputa em questão, homens se encontram no extremo de dominância.

A violência simbólica é uma forma de coação que não é física. É uma dor que pode ser social, psicológica e/ou emocional. É considerada também um constrangimento, um desconforto ou estranhamento. O conceito de violência simbólica aponta de que maneira a dominância (autoridade e poder de pessoas/instituições) é naturalizada, ou seja, consideradas normais em uma sociedade (VASCONCELOS, 2002).

Nos relatos, Júlia e Liz narram sentir violência simbólica cotidianamente. A dominação masculina é a naturalização da superioridade do homem sobre a mulher. Então, observa-se que o homem detém mais capital do que a mulher no campo científico de exatas. Quando as mulheres conquistam esses capitais e ocupam esses espaços ocorre a violência simbólica contra elas.

O somatório dos eventos de violência simbólica, advinda da disputa de poder no campo científico, está relacionada a consequências na saúde mental das pesquisadoras - como a ansiedade generalizada e a síndrome do impostor. Toda 
essa disputa no dia a dia também leva a um alto índice de abandono de mulheres nas exatas.

\title{
Hierarquização de mulheres nas ciências exatas
}

\begin{abstract}
"A trajetória da mulher brasileira nos últimos séculos é, para dizer pouco, extraordinária: de uma educação no lar e para o lar, no período colonial, para uma participação tímida nas escolas públicas mistas do século 19; depois, uma presença significativa na docência do ensino primário, seguida de uma presença hoje majoritária em todos os níveis de escolaridade, bem como de uma expressiva participação na docência da educação superior.” (GROSSI et al., 2016)
\end{abstract}

A dificuldade em encontrar mulheres em posições seniores está presente nos relatos da Júlia e Liz. Mesmo com uma trajetória de conquistas do último século, observa-se dificuldade de hierarquização de mulheres na carreira científica - especialmente no que diz respeito a posições de destaque, reconhecimento ou liderança (mecanismo social "teto de vidro").

De acordo com o CNPq (Conselho Nacional de Desenvolvimento Científico e Tecnológico), o número de cientistas mulheres é equivalente ao de homens em sua base de dados. O cenário muda para bolsistas de produtividade: no sistema de classificação do CNPq, pesquisadoras são encontradas em níveis mais baixos. Ao mesmo tempo em que os pesquisadores do sexo masculino são encontrados com mais frequência em níveis altos (1A e 1B), especialmente nas áreas de 'Engenharia, Ciências Exatas, Ciências da Terra' e 'Ciências da Vida'. Quando avaliada apenas as ciências humanas e sociais essa diferença não foi encontrada (VALENTOVA et al., 2017). Esta bolsa de produtividade funciona como um capital científico importante (conceito bourdieusiano), já que a classificação de produtividade favorece maior financiamento de pesquisa para posições superiores. Como mais homens estão atualmente presentes nessas posições mais altas, há uma retroalimentação do ciclo de menor presença de produtividade de mulheres na ciência.

Outro exemplo da ausência de mulheres na liderança é o próprio CNPq, que nunca teve uma presidente mulher. Além do CNPq, a Academia Brasileira de Ciências, entidade que reúne cientistas eminentes do Brasil nos campos de 
Ciências Matemáticas, Físicas, Químicas, da Terra, Biológicas, Biomédicas, da Saúde, Agrárias, da Engenharia e Sociais: atualmente tem-se 467 homens titulares diante de 86 mulheres, representando 16\% do total. A instituição tem mais de um século de idade, mas nunca teve uma mulher no cargo de presidência (ACADEMIA BRASILEIRA DE CIÊNCIAS, 2020). Apesar de mulheres representarem metade da força de trabalho científico, também nunca houve uma ministra efetiva no Ministério da Ciência, Tecnologia e Inovações (BRASIL, 2020).

Ao investigar a presença e áreas de interesse de brasileiras com teses de doutorado defendidas entre 2000 e 2013, observou-se uma participação feminina maior nas áreas das Ciências Biológicas, das Ciências da Saúde e das Ciências Humanas, sendo que a menor participação se dá nas Engenharias (GROSSI et al., 2016). Especificamente, a presença de mulheres na área de exatas é pequena e diminui desproporcionalmente ao passo que se avança na carreira. Dessa maneira, existe sub-representação dentro da área de conhecimento e em relação à hierarquização (BARBOSA; LIMA, 2013). Para compreender esses fatos, aspectos históricos da inserção das mulheres na área de exatas podem ser úteis.

Registros apontam que a primeira brasileira a se formar em direito foi em 1888 e a primeira a se formar medicina foi 1882. A estreia de mulheres em exatas aconteceu com pelo menos duas décadas de atraso se comparado com as áreas da saúde e direito. A primeira mulher a se formar em Engenharia foi em 1919 seguido por um vácuo até 1926, quando mais uma mulher tornou-se engenheira. As mulheres passam a ser aceitas na Escola Politécnica somente em 1928 (QUEIROS, 2001). Já na física, tem-se mais de uma década de atraso em relação às primeiras engenheiras.

Os primeiros doutores obtiveram seus títulos no exterior, o que inviabilizou a mesma oportunidade para as mulheres devido ao fato de uma jovem viajar sozinha não ser apropriado de acordo com o habitus do séc. XX. O ingresso tardio das mulheres no Ensino Superior é um dos fatores históricos que 
explicam a demora da inserção delas na nas áreas de exatas, com influência até hoje em todos os estágios da carreira (BARBOSA; LIMA, 2013).

Diante da invisibilização das mulheres no dia a dia de Júlia e Liz, assim como a ausência de referências de mulheres que chegaram a conquistar um cargo profissional em suas áreas de atuação, a consequência é o constante contato com a vontade de desistir (Liz) e a efetivação da desistência (Júlia). Ambas relatam que sua permanência na carreira de análise de dados está amplamente relacionada com as redes sociais de apoio femininas de apoio que conquistaram e mantiveram ao longo do tempo.

\section{Estratégias de enfrentamento: Redes de apoio}

O uso de estratégias de enfrentamento é definido como o empenho de esforços cognitivos e comportamentais como alternativa para minimizar, tolerar, aceitar, demandas internas e/ou externas com potencial estressor ou que leve a diminuição da qualidade de vida e bem estar mental. Essas redes sociais de apoio são formadas pelo conjunto de sistemas e de pessoas, que compõem os elos de relacionamento recebidos e percebidos. são uma estratégia de enfrentamento indireto e proporcionam portanto um aumento da capacidade de enfrentamento de situações adversas da mais distinta ordem (JULIANO, 2014; NETTO et al., 2017; ROSA, 2009) .

Na vivência de mulheres essas redes sociais de apoio feminina ganham força e destaque, pois, são uma das principais estratégias para fornecimento de suporte emocional, planejamento do enfrentamento de problemas (NETTO et al., 2017). De acordo com os depoimentos de Júlia e Liz, essa demanda existe e é de extrema importância na área das exatas, ambas apresentaram em algum nível o desenvolvimento de condições mentais patológicas como síndrome do impostor e TAG (Transtorno de Ansiedade Generalizada).

As dificuldades emocionais representadas aqui pelas pesquisadoras se estendem a mulheres de outras áreas - o que evidencia que esses agravamentos não são apenas uma dificuldade específica da área de atuação delas. De acordo com a 
Organização Mundial de Saúde, transtornos de ansiedade acometem uma a cada três pessoas no mundo - equivalente a $4 \%$ da população mundial (ORGANIZAÇÃO MUNDIAL DE SAÚDE, 2017). Entre as mulheres esse índice é em torno de $42 \%$ enquanto que para homens está em torno de $29 \%$. Já a síndrome do impostor apresenta uma maior tendência para acontecer entre jovens mulheres quando comparadas com homens (BRAVATA et al., 2020). Também é importante perceber que essas condições estão frequentemente associadas ao transtorno depressivo (ORGANIZAÇÃO MUNDIAL DE SAÚDE, 2017).

Dadas essas condições de desenvolvimento de distúrbios psicológicos, percebese uma diminuição da capacidade produtiva de mulheres. Além desse decaimento, há um aumento de prejuízos cognitivos levando a uma menor qualidade de vida. Durante o isolamento social ocorrido em virtude da pandemia de COVID-19, onde o contato social ficou restrito, essas redes sociais de apoio ganharam evidência através de redes sociais (digitais) o que tem auxiliado na superação das adversidades impostas. Júlia e Liz reforçam o papel essencial dessas redes sociais de apoio feminina nas suas produtividades e bem estar.

Ao avaliar a conjuntura a qual as mulheres estão envolvidas com subvalorização de seus feitos profissionais e uma jornada dupla em suas rotinas percebe-se a urgente necessidade de melhorar os ambientes profissionais e domésticos ocupados por mulheres, e que um caminho promissor é os fortalecimentos destas redes sociais de apoio femininas.

\section{Considerações finais}

Esse relato de experiência evidenciou a presença, ainda muito forte mesmo que se trate do ano de 2020, da violência simbólica no dia a dia de pesquisadoras atuantes na área de exatas. Essa violência contribuiu para o desenvolvimento de transtornos como ansiedade generalizada e a síndrome do impostor. Um aspecto relevante para a experiência das pesquisadoras é a ausência de mulheres em posições altas da carreira, ou seja, existem mulheres se 
qualificando, mas a presença de mulheres orientando, recebendo prêmios e conquistando vagas de liderança é ínfima. A união do desgaste emocional do dia a dia no trabalho somado à ausência de mulheres de sucesso em suas áreas ocasionou num histórico de desistência. No entanto, os depoimentos não são casos de insucesso.

As pesquisadoras encontraram uma estratégia de enfrentamento em comum: Redes sociais de apoio femininas. Suas redes, formadas por outras mulheres, proporcionam um ambiente seguro de troca, de apoio emocional e de confiança por não se sentirem sozinhas num ambiente de trabalho hostil. Em meio a pandemia da COVID-19, essa rede social de apoio continua tendo um papel essencial, especialmente pelo aumento de abalos emocionais em decorrência dela.

É nítida a necessidade e importância dessas redes para enfrentamento de situações adversas, para a promoção de resiliência e para a permanência de mulheres na ciência. Consequentemente, pode-se esperar que sua permanência seja benéfica para o aumento do interesse de outras mulheres na área auxiliando a escrever uma nova história em lugar da ausência e invisibilização histórica sofrida por mulheres nas ciências exatas.

\section{Referências}

ACADEMIA BRASILEIRA DE CIÊNCIAS. Membros - ABC. Disponível em: $<$ http://www.abc.org.br/membros/>. Acesso em: 21 jul. 2020.

BARBOSA, M. C.; LIMA, B. S. Mulheres na Física do Brasil: Por que tão poucas? E por que tão devagar? In: Livro Trabalhadoras, 2013.

BRASIL. Ministério da Ciência, Tecnologia e Inovação. Disponível em: <http://www.mctic.gov.br>. Acesso em: 27 jul. 2020.

BRAUER, K.; PROYER, R. T. Are Impostors playful? Testing the association of adult playfulness with the Impostor Phenomenon. Personality and Individual Differences, v. 116, p. 57-62, Oct. 2017.

BRAVATA, D. M. et al. Prevalence, predictors, and treatment of impostor syndrome: a systematic review. Journal of General Internal Medicine, v. 35, n. 4, p. 1252-1275, Apr. 2020.

BRUSCHINI, M. C. A. Estudos sobre mulher e educação: algumas questões sobre o magistério. Cadernos de Pesquisa, v. 64, p. 4-13, 1988.

COELHO, D.; DE NEGRI, J. A.; DE NEGRI, F. Ascensão profissional de homens 
e mulheres nas grandes empresas brasileiras. In: Tecnologia, exportação e emprego. Brasília: Instituto Econômico de Pesquisa Aplicada, 2006.

CORTÉS, O. N. P. A inter-relação Bourdieusiana: habitus, campo e capital. Dissertação de mestrado-[s.l.] Pontifícia Universidade Católica do Rio Grande do Sul, 2016.

DAVIDSON, M. Shattering The Glass Ceiling: The Woman Manager. London: Paul Chapman, p. 192, 1992.

GROSSI, M. G. R. et al. As mulheres praticando ciência no Brasil. Revista Estudos Feministas, v. 24, n. 1, p. 11-30, Apr. 2016.

INTERNATIONAL MATHEMATICAL UNION (IMU). Fields Medal. Disponível em: <https://www.mathunion.org/imu-awards/fields-medal>. Acesso em: 15 jul. 2020.

JULIANO, M. C. C. Reflexões sobre rede de apoio social como mecanismo de proteção e promoção de resiliência. Ambiente \& Sociedade, v. XVII, n. 3, p. 135-154, Sep. 2014.

KOLLIGIAN, J.; STERNBERG, R. J. Perceived fraudulence in young adults: is there an "imposter syndrome"? Journal of personality assessment, v. 56, n. 2, p. 308-326, Apr. 1991.

MADALOZZO, R. CEOs e composição do conselho de administração: a falta de identificação pode ser motivo para existência de teto de vidro para mulheres no Brasil? Revista de Administração Contemporânea, v. 15, n. 1, p. 126-137, Feb. 2011.

MAY, T. Pesquisa social: questões, métodos e processos. Tradução Carlos Alberto Silveira Netto Soares. 3. ed. Porto Alegre: Artmed, 2004.

MILKMAN, K. L.; AKINOLA, M.; CHUGH, D. What happens before? A field experiment exploring how pay and representation differentially shape bias on the pathway into organizations. The Journal of applied psychology, v. 100, n. 6, p. 1678-1712, nov. 2015.

MINAYO, M. C. DE S. Análise qualitativa: teoria, passos e fidedignidade. Ciência \& Saúde Coletiva, v. 17, n. 3, p. 621-626, Mar. 2012.

MOSS-RACUSIN, C. A. et al. Science faculty's subtle gender biases favor male students. Proceedings of the National Academy of Sciences of the United States of America, v. 109, n. 41, p. 16474-16479, 9 Oct. 2012.

NETTO, L. DE A. et al. Social support networks for women in situations of violence by an intimate partner. Texto \& Contexto - Enfermagem, v. 26, n. 2, 2017.

ORGANIZAÇÃO MUNDIAL DE SAÚDE. Depression and Other Common Mental Disorders: Global Health Estimates. [s.l.] OMS, 2017. 
ORGANIZAÇÃO PRÊMIO ABEL. Laureates Abel Prize. Disponível em: <https://www.abelprize.no/c53673/seksjon/vis.html?tid=53719>. Acesso em: 15 jul. 2020.

ORGANIZAÇÃO PRÊMIO NOBEL. All Nobel Prizes. Disponível em: $<$ https://www.nobelprize.org/prizes/lists/all-nobel-prizes>. Acesso em: 13 jul. 2020.

PARENT IN SCIENCE. Produtividade acadêmica durante a pandemia: Efeitos de gênero, raça e parentalidade. Disponível em: <https://327b6o4e-5cf4$492 b-910 b-$ e35e2bc67511.filesusr.com/ugd/ob341b_81cd839odof94bfd8fcd17ee6f29bcoe. pdf?index=true $>$. Acesso em: 19 jul. 2020.

QUEIROS, D. M. Raça, gênero e educação superior.Tese de doutorado-2001.

RISSO, C. DE A. «Gosto» é discutível: uma reflexão sobre o acúmulo de bens simbólicos. Matrizes, v. 2, n. 1, 2008.

ROSA, T. E. DA C. As redes sociais e de apoio: o conviver e a sua influência sobre a saúde. Boletim do Instituto de Saúde, 2009.

STEINPREIS, R. E.; ANDERS, K. A.; RITZKE, D. The Impact of Gender on the Review of the Curricula Vitae of Job Applicants and Tenure Candidates: A National Empirical Study. Springer Science and Business Media LLC, 1999.

VALENTOVA, J. V. et al. Underrepresentationofwomen in the senior levelsofBrazilianscience. PeerJ, v. 5, p. e4000, 19 Dec. 2017.

VASCONCELOS, M. D. Pierre Bourdieu: A herança sociológica. Educação \& Sociedade, v. 23, n. 78, p. 77-87, Apr. 2002.

WENNERAS, C.; WOLD, A. Nepotism and sexism in peer-review. Nature, v. 387, n. 6631, p. 341-343, 22 May 1997.

WORLD ECONOMIC FORUM. Global Gender Gap Report 2020. Suíça: World Economic Forum, 2019.

WRIGHT, E. O.; BAXTER, J. The glass ceiling hypothesis. v. 14, n. 6, p. 814821, Dec. 2000. 